\title{
Development of CRISPR for Molecular Diagnostics and Gene Therapy
}

\author{
Esther Pak and Martin P Stewart* \\ UTS School of Life Sciences, Australia \\ *Corresponding author: Martin P Stewart, UTS School of Life Sciences, Australia
}

\begin{tabular}{|c|c|}
\hline ARTICLE INFO & ABSTRACT \\
\hline Received: 幽 December 31, 2020 & $\begin{array}{l}\text { Molecular diagnostics is crucial to the identification and control of diseases. Rapid } \\
\text { and accurate diagnosis directly supports the implementation of public health measures }\end{array}$ \\
\hline Published: 慧 January 15, 2021 & to limit infection and drive the development of corresponding vaccines and therapeutics. \\
\hline $\begin{array}{l}\text { Citation: Esther Pak, Martin P Stewart. } \\
\text { Development of CRISPR for Molecular } \\
\text { Diagnostics and Gene Therapy. Biomed } \\
\text { J Sci \& Tech Res 33(1)-2021. BJSTR. } \\
\text { MS.ID.005357. }\end{array}$ & $\begin{array}{l}\text { of the state of diagnostics in the public health sphere. CRISPR, an RNA-guided gene-editing } \\
\text { tool with the ability to find and edit desired DNA sequences, has recently emerged and } \\
\text { gained further momentum amidst the COVID-19 pandemic. Compared to the current PCR } \\
\text { test, the faster reaction time, result turnover and cheaper cost has highlighted CRISPR's } \\
\text { potential as a point-of-care diagnostic tool that offers faster results and the possibility of } \\
\text { at-home testing. Beyond diagnosis, CRISPR is a genome editing technology with the ability } \\
\text { to cut, paste and modify specific DNA sequences, which has the future potential to find } \\
\text { and possibly eliminate the virus on the molecular level. The rapidly evolving nature of the } \\
\text { SARS-CoV-2 virus and threat of future coronavirus pandemics provides further incentive } \\
\text { to explore CRISPR-based diagnostics and gene therapy. Herein, the review will discuss } \\
\text { the current state of molecular diagnostics in the context of the health response to the } \\
\text { COVID-19 pandemic and explore the benefits and concerns of CRISPR as an alternative } \\
\text { diagnostic tool and its future directions in gene therapy. }\end{array}$ \\
\hline
\end{tabular}

\section{Mini Review}

The coronavirus disease (COVID-19) pandemic caused by the severe acute respiratory syndrome coronavirus.

2 (SARS-CoV-2 virus) continues to pose a global health risk, with over 30 million confirmed cases and almost [1] million deaths, as of September 19, 20201. Despite the development of tests for diagnosing the disease, testing is still minimal in comparison to the extent of the outbreak, due to the limited diagnostic capacity of the PCR [2]. This poses a significant problem, as widespread testing and adopting subsequent monitoring strategies were key to containing the spread of the disease in successful countries like Germany and South Korea [3].

CRISPR (clustered regularly interspaced short palindromic repeats) is a powerful and versatile gene editing tool derived from a naturally occurring bacterial immune response [4]. Consisting of two components, a guide RNA that can recognise a target DNA sequence and a Cas protein that can cut DNA, CRISPR acts as a pair of molecular scissors with the ability to quickly find, cut and paste desired areas of the genome by precisely changing any portion of DNA [5]. Despite being primarily thought of as a gene-editing tool, both gene detection and editing are possible through the one technology, as a signal is released once the CRISPR-Cas system finds and cuts its target [5]. More so than their PCR counterparts for the diagnosis of COVID-19, CRISPR-based molecular diagnostics is highly sensitive with the ability to test the same sample multiple times, faster in producing results and even has the potential to adapt to new targets [6]. Moreover, the specific design of the CRISPR-based tests for point-of-care settings like hospitals allow for the tests to be much more widespread and cost-effective than the currently used PCR, addressing the key problem associated with the current means of testing [6].

While much research has been focused on the development of CRISPR-Cas systems for diagnosis of disease, there has also been great interest in the gene therapy aspect of the technology. 
By harnessing the genome-editing ability of the CRISPR-Cas13 system, there has been development of an antiviral strategy called PAC-MAN (prophylactic antiviral CRISPR in human cells) using CRISPR RNAs (crRNAs), which has been found to target $90 \%$ of coronaviruses and shown great potential to act as a viral inhibitor for future coronavirus and influenza threats [7]. Despite this being a favourable future direction, CRISPR diagnostics alone is not yet ready for clinical use, with further research being required to maximise the safety and efficacy of both diagnosis and gene-editing of COVID-19 and future diseases.

\section{The Need for An Alternative to PCR}

Over the past several decades, there have been recurring epidemics of a large scale due to various emerging viruses, including HIV, Middle East Respiratory Syndrome Coronaviruses (MERS), Zika, and the current SARS-CoV-2 [8]. A common factor of these epidemics that significantly contributed to their severity and duration is the lack of a rapid and accurate means of molecular diagnostics, which has continually inhibited the public health response and the ability to properly screen for and control viral threats [9]. With the SARS-CoV-2 virus causing over 30 million confirmed cases and almost 1 million deaths as of September 19,2020 , accelerating the rate and scale of testing, especially to limit the risk of asymptomatic carriers spreading the disease, has become an urgent health concern on a global scale $[1,10]$.

The current gold standard for testing of COVID-19 are assays that use quantitative real time polymerase chain reaction (qRT-PCR) [10]. This process involves sample collection and transport, RNA extraction, reverse transcription and amplification10. However, the extremely high demand, paired with the slow process of delivering the samples to centralised laboratories for testing, has made 2472 hours the standard wait time for receiving results [11]. As well as this, the test itself requires extensive human labour in taking several hours to complete, as well as expensive equipment and reagents that are at risk of potential shortages. Furthermore, the limited information provided by the qRT-PCR results is arguably the most restrictive aspect of the testing method, only being able to indicate whether the virus is present at the time of sample collection [6]. As such, the test provides no further insight into the patient's past infection or possible development of immunity and may even potentially deliver false results to patients in early-stage infection who may show symptoms after the test is taken [6].

\section{Isothermal Amplification}

The main factors preventing more widespread testing of the SARS-CoV-2 virus include the long results processing times and high demand that continues to overwhelm healthcare systems, which are limited to the current means of qRT-PCR testing [6]. The whole testing process takes between several hours to several days to produce results, primarily due to the lengthy duration of thermal cycling required in the PCR testing process [10]. Isothermal amplification, specifically the reverse transcription loop-mediated isothermal amplification (RT-LAMP_ of nucleic acids presents an alternative to PCR, as it allows for extreme amplification of nucleic acids at constant temperatures to skip the thermal cycling process altogether [12]. Avoiding this process significantly decreases the cost and complexity of the diagnostic device, effectively reducing the time needed to process test results [6]. As the amplified DNA can also be simply detected through dyes that visually indicate the change in turbidity, isothermal amplification demonstrates great potential as an alternative method to PCR due to its simplicity of understanding results, much faster amplification time and potential to test multiple samples at once [13]. The main limitations of isothermal amplification, however, include optimising the conditions of the reaction and creating sequence-specific primers [14]. To address this problem of non-specific amplification, isothermal amplification must be combined with a detection method for specific genes. A viable solution for this specific gene detection is offered by the highly specific CRISPR-Cas protein technology [15].

\section{CRISPR for Molecular Diagnostics}

CRISPR is an extremely versatile and powerful gene-editing tool that has demonstrated promising therapeutic results in clinical trials in recent years [16]. Part of its ability to act as a pair of molecular scissors is its capacity to find and detect specific nucleic acids in any given sample with its associated Cas proteins [15]. Instead of its use for genome editing, the COVID-19 pandemic has generated impetus for the use of CRISPR as a molecular diagnostic tool to create more rapid, accurate and inexpensive tests to detect infectious viral diseases [9]. Recently, a special group of Cas proteins, including Cas12 and Cas13, was found to have the RNA detection abilities [17]. Several new lateral-flow assays have been developed as molecular diagnostic tools for the detection of SARS-CoV-2 viral RNA at a rapid speed, with the combination of CRISPR and isothermal amplification [17]. Notably, two biosciences companies - Mammoth Biosciences and Sherlock Biosciences - have been at the forefront of the CRISPR-based diagnostics race9. While Mammoth has created a DNA endonuclease-targeted CRISPR trans reporter (DETECTR) system that is able to detect multiple strains of the coronavirus, Sherlock has developed specific high-sensitivity enzymatic reporter unlocking (SHERLOCK) [10]. The main advances of these systems over PCR are their greater sensitivity, ability to multiplex numerous samples at once, visual readout and quantitative detection [10]. Both these systems utilise the CRISPR nucleases that are programmed to find a specified DNA sequence without the need for complex and time-consuming lab protocols [9]. SHERLOCK harnesses the cleavage and degrading of neighbouring ssRNA by the Cas13 proteins to cleave and activate a fluorescent reporter, while DETECTR uses SSDNA and Cas12a. Once the desired DNA sequence is found, the nuclease generates a fluorescent signal after cutting a reporter DNA strand from the sample, confirming 
the identification of the viral sequence [9]. After RNA is extracted, both systems require pre-amplification of DNA or RNA before the results are able to be revealed through a lateral-flow assay. This process is illustrated in Figure 1a, where the DETECTR system conducts reverse transcription and isothermal amplification using RT-LAMP, previously discussed as a viable alternative to the current RT-PCR [10]. The main weakness of RT-LAMP in performing non- specific amplification is then addressed by the use of the CRISPR protein Cas12a's detection of a selected coronavirus viral sequence, which is then cuts the reporter molecule to confirm the presence of the virus [10]. The final step is the displaying of results, which are simply visualised on a lateral-flow strip, pictured in Figures $1 \mathrm{~b}$ \& 1c.

a)

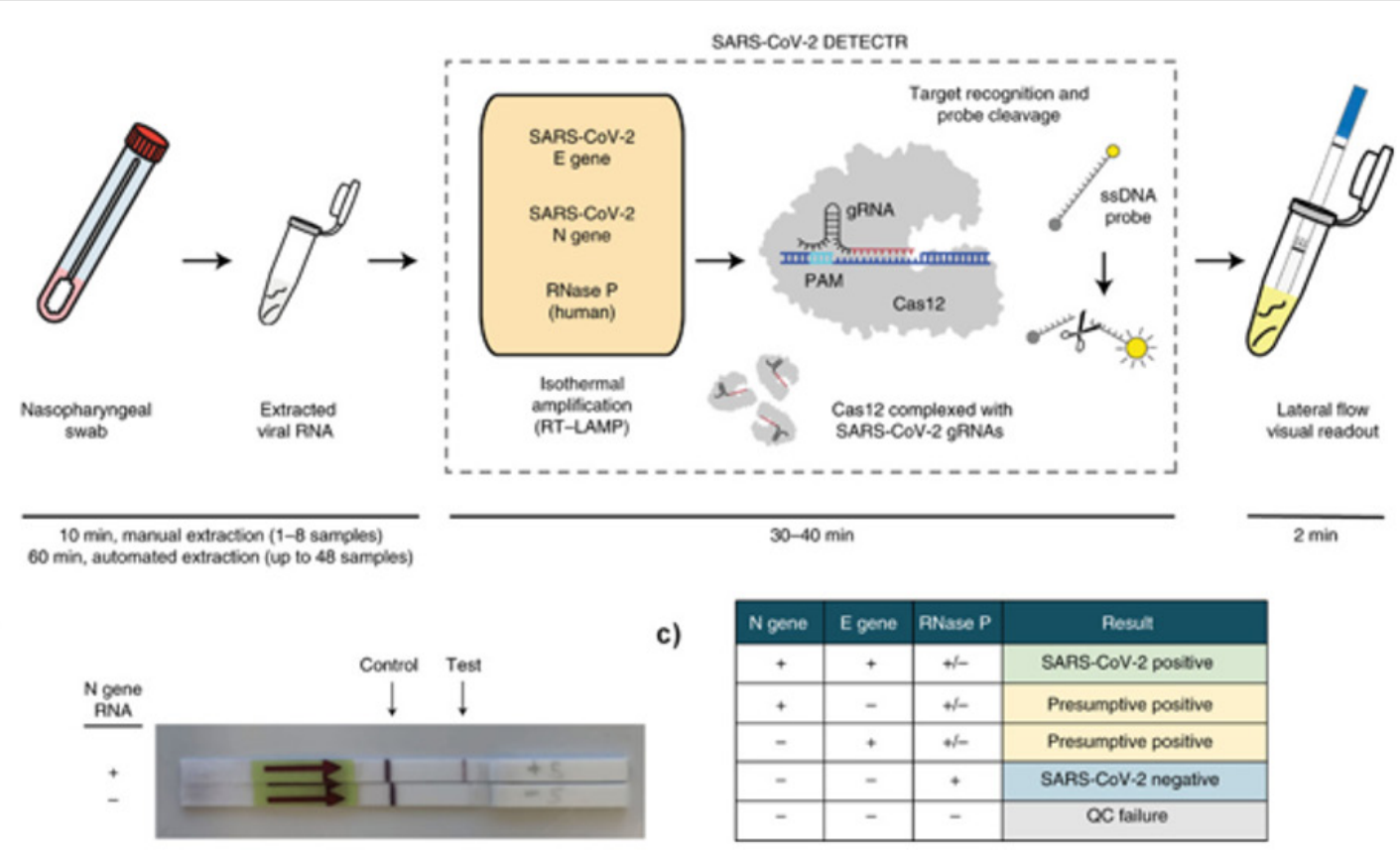

Figure 1:

a) Diagram of the steps involved in the process of CRISPR-based molecular diagnostics using the SARS-CoV-2 DETECTR system. Conventional sample collection is conducted using a nasopharyngeal swab. RNA is extracted from the sample. This is followed by reverse transcription loop-mediated isothermal amplification (RT-LAMP) and Cas12-based detection of target genes. The results of the DETECTR are visually presented on a lateral-flow strip, similar to a pregnancy test, for quantitative analysis of results.

b) Photograph of a lateral-flow strip assay read-out. The control and test lines are indicated.

c) Interpretation of results: a positive result requires the detection of at least the two SARS-CoV-2 viral gene targets (N gene and E gene). (Santiago, 2020).

Table 1: Comparison of the qRT-PCR assay and DETECTR assay for the detection of SARS-CoV-2 Broughton et. [9].

\begin{tabular}{|c|c|c|}
\hline & qRT-PCR assay & DETECTR assay \\
\hline Target Gene & E gene and $\mathrm{N}$ gene & N gene (N1, N2 and N3) \\
\hline Sample Control & RNase P & RNase P \\
\hline Limit of Detection & 1-3.2 copies per $\mu$ input & 10 copies per $\mu$ input \\
\hline Approximate Reaction Time & 2 hours & 30-40 minutes \\
\hline Approximate Sample-to-Result Time & 4 hours & 45 minutes \\
\hline Assay Results Type & Qualitative & Quantitative \\
\hline Assay Components & $\begin{array}{c}\text { UDG digestion }\left(25^{\circ} \mathrm{C}, 2 \mathrm{~min}\right) \text {, reverse } \\
\text { transcription }\left(50^{\circ} \mathrm{C}, 15 \mathrm{~min}\right) \text {, denaturation } \\
\left(95^{\circ} \mathrm{C}, 2 \mathrm{~min}\right) \text {, amplification }\left(95^{\circ} \mathrm{C}, 3 \mathrm{~s} ; 55^{\circ} \mathrm{C}, 30 \mathrm{~s} ;\right. \\
45 \text { cycles })\end{array}$ & $\begin{array}{l}\text { RT- } \operatorname{CaMP}\left(63^{\circ} \mathrm{C}, 20-30 \mathrm{~min}\right), \\
\text { Lateral flow strip (RT, } 2 \text { min; no additional time } \\
\text { with fluorescence readout) }\end{array}$ \\
\hline Bulky Instrumentation Required & Yes & No \\
\hline
\end{tabular}


This entire assay is able to run in approximately 30 minutes, specifically identifying the SARS-CoV-2 virus with a sensitivity quite similar to the standard RT-PCR [9]. The use of microfluidic chips to increase the testing capacity would further improve the DETECTR platform as a diagnostic tool capable of multiplexing thousands of samples simultaneously [18]. CRISPR-based diagnostics is able to achieve this level of efficiency because of its own characteristic selectivity of DNA sequences, the high sensitivity of molecular diagnostics, and the simplicity of the lateral-flow assays that are also used in at-home pregnancy tests [9]. The main benefits of CRISPR over the conventional RT-PCR are its adaptability to new targets that offers rapid reading of results, as well as its ability to run the same sample several times to decrease the likelihood of false negative results, further outlined in Table 1 [19]. With its use of cheaper materials and faster results turnover, the tests are being further developed for use in a point-of-care setting, to increase the scale of screening and accessibility from an easier at-home testing scenario [12]. However, it is predicted that there are several months before the testing procedure is finalised regulatory approval is obtained, and the test is able to go into mass scale production with the implementation of new protocols [19].

\section{CRISPR for Gene Therapy}

While CRISPR shows great potential as a molecular diagnostic tool, especially in the midst of the viral COVID-19 pandemic, future applications of the technology in gene therapy are of equal or even greater importance to the future health responses to viral outbreaks and treatment of disease [7]. Multiple strains of coronavirus have emerged and been transmitted from animal reservoirs to humans in the last two decades, causing the SARS, MERS and current COVID-19 diseases, which have resulted in significant fatalities and an ongoing global pandemic [20]. With a recent report revealing the circulation of two new strains of the SARS-CoV-2 virus with varying genomic sequences that indicate the virus' evolution, there is a pressing need to develop methods not limited to just curing SARSCoV-2 but to broadly defend against other future coronaviruses [21]. The lifecycle of the SARS-CoV-2 virus is likely to be similar to the related coronavirus caused by SARS, where the virus enters the cell, releases its RNA genome, from which negative-sense RNAs are synthesised to produce viral mRNAs and new copies of the positive-sense viral genome [22]. While current vaccine trials work through priming the human immune system to recognise the coronavirus proteins and decrease the initial entry of the virus into the cell, the antiviral approach being developed relies on CRISPR to recognise and degrade the viral genome and its viral mRNA copies that spread the virus in the body [23]. By selectively targeting the positive-sense genome with CRISPR-Cas $13 \mathrm{~d}$, it is expected that the protein can inhibit viral function and replication by cutting all the positive-sense viral RNA [7]. The Cas-13d protein was chosen over other proteins for its high specificity and catalytic activity that showed the potential to most efficiently identify and destroy RNA viruses, such as SARS-CoV-2 and the influenza A virus (IAV) [24]. The development of the PAC-MAN strategy was aimed to target multiple species of coronaviruses to provide protection against future emerging strains of the disease [7].

The testing of PAC-MAN as an antiviral strategy revealed a group of six crRNAs to be able to target $91 \%$ of coronavirus sequences and $92 \%$ of 91,600 influenza strains [25]. This system of using crRNA to target different coronaviruses or different regions of the same coronavirus could be a potential antiviral strategy to protect against viral evolution and the likely possibility of new strains of the virus [7]. As such, the application of the CRISPR-Cas systems show promising applications beyond diagnostics of diseases, such as the previously mentioned DETECTR and SHERLOCK molecular diagnostic systems, and into the field of gene therapy to disable the virus at the molecular level [7]. Whilst initial studies have shown relative success of PAC-MAN as a prophylactic system used defensively before new viral outbreaks, further examinations should be made regarding its ability to reduce viral genetic material upon infection [7]. Another key consideration to be made regarding the PAC-MAN strategy is its current testing having occurred in vitro, with synthesised SARS-CoV-2 fragments and live infection using an H1N1 IAV strain in human lung cells [26]. Further exploration of PAC-MAN's ability to be delivered into human respiratory tract cells and inhibit viruses in vivo must be made to investigate its viability for clinical trials and therapeutic use [7]. Unlike the use of CRISPR for molecular diagnostics, which has the potential to begin mass production and implementation in a matter of months [27], CRISPR's use in gene therapy through strategies like PAC-MAN will likely take years to be deployed in a clinical setting [7]. Hence, though PAC-MAN will not be able to aid the current COVID-19 pandemic, there is promising potential for its use against the viral threats likely to emerge in the future [7].

\section{Conclusion and Outlook}

In conclusion, the development of CRISPR as a molecular diagnostic tool and potential gene therapy shows a promising future for the medical field. The current use of the qRT-PCR lacks the testing capacity and speed to allow for widespread population testing to properly screen and combat global health pandemics like COVID-19. However, the use of isothermal amplification to increase test result turnover, paired with the selectivity and specificity of CRISPR, has allowed for the creation of systems such as DETECTR and SHERLOCK. With the speed gained from avoiding the timeconsuming process of PCR heat cycling and the simplicity and low cost of lateral-flow assays, it is likely to be a matter of months before this much more effective form of testing is mass produced and implemented to help with the testing of larger samples of the population at once. Further developments in the technology to allow for point-of-care and at-home testing should be of high priority, to tackle the problem of slow test result turnover and equipment 
and reagent supply shortages of the conventional PCR test. On the other hand, the traditional usage of CRISPR for genome editing has been developed into the PAC-MAN antiviral strategy. Gene therapy against viral threats as a pan-coronavirus defence mechanism using CRISPR, though being a promising field of research, requires further research and development into effective and safe in vivo delivery methods of the PAC-MAN into human cells.

\section{Acknowledgement}

We gratefully acknowledge Sushil Kandel, who aided in the development of this review.

\section{References}

1. (2019) World Health Organization. WHO coronavirus disease (COVID-19) dashboard.

2. Roser M, Ritchie H, Ortiz-Ospina E, Hasell J (2020) Coronavirus pandemic (COVID-19).

3. Normile D (2020) Coronavirus cases have dropped sharply in South Korea. What's the secret to its success? Science.

4. Pan A, Kraschel K (2018) CRISPR diagnostics: underappreciated uses in perinatology. Seminars in Perinatology 42(8): 525-530.

5. Daley J (2020) CRISPR gene editing may help scale up coronavirus testing. Scientific American.

6. Santiago I (2020) Trends and innovations in biosensors for COVID-19 mass testing. ChemBioChem.

7. Abbott T, Dhamdhere G, Liu Y, Lin X, Goudy L, et al. (2020) Development of CRISPR as an antiviral strategy to combat SARS-CoV-2 and influenza. Cell 181: 865-876.

8. Wang C, Horby P, Hayden F, Gao G (2020) A novel coronavirus outbreak of global health concern. Lancet 395: 470-473.

9. Broughton J, Deng X, Yu G, Fasching C, Servellita V, et al. (2020) CRISPRCas12-based detection of SARS-CoV-2. Nature Biotechnolology 38: 870874.

10. Bai Y, Yao L, Wei T, Tian F, Jin D, et al. (2020) Presumed asymptomatic carrier transmission of COVID-19. JAMA 323(14): 1406-1407.

11. Zhang W, Du R, Li B, Zheng X, Yang X, et al. (2020) Molecular and serological investigation of 2019-nCoV infected patients: implication of multiple shedding routes. Emerging Microbes \& Infections 9(1): 386389.

\section{ISSN: 2574-1241}

DOI: 10.26717/BJSTR.2021.33.005357

Martin P Stewart. Biomed J Sci \& Tech Res

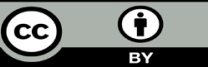

This work is licensed under Creative Commons Attribution 4.0 License

Submission Link: https://biomedres.us/submit-manuscript.php
12. Esbin MN, Whitney ON, Chong S, Maurer A, Darzacq X, et al. (2020) Overcoming the bottleneck to widespread testing: a rapid review of nucleic acid testing approaches for COVID-19 detection. RNA 26(7): 771-783.

13. Mori Y, Notomi T (2009) Loop-mediated isothermal amplification (LAMP): a rapid, accurate, and cost-effective diagnostic method for infectious diseases. J Infect Chemotherapy 15(2): 62-69.

14. Craw P, Balachandran W (2012) Isothermal nucleic acid amplification technologies for point-of-care diagnostics: a critical review. Lab on a Chip 12(14): 2469-2486.

15. Adli M (2018) The CRISPR tool kit for genome editing and beyond. Nature Communications 9(1): 1911.

16.Jiang F, Doudna J (2017) CRISPR-Cas9 Structures and Mechanisms. Annual Review of Biophysics 46: 505-529.

17. Gootenberg J, Abudayyeh O, Lee J, Essletzbichler P, Dy A, et al. (2017) Nucleic acid detection with CRISPR-Cas13a/C2c2. Science 356(6336): 438-442.

18. Ackerman CM, Myhrvold C, Thakku SG, Freije CA, Metsky HC, et al. (2020) Massively multiplexed nucleic acid detection with Cas13. Nature 582(7811): 277-282.

19. Service R (2020) The standard coronavirus test, if available, works well - but can new diagnostics help in this pandemic?

20. Shi Z, Hu Z (2008) A review of studies on animal reservoirs of the SARS coronavirus. Virus Research, 133(1): 74-87.

21. Tang X, Wu C, Li X, Song Y, Yao X, et al. (2020) On the origin and continuing evolution of SARS-CoV-2. National Science Review 7(6): 1012-1023.

22. Lanying D, He Y, Zhou Y, Liu S, Zheng B, et al. (2009) The spike protein of SARS-CoV - a target for vaccine and therapeutic development. Nature Reviews Microbiology 7: 226-236.

23. Rappuoli R (2018) Glycoconjugate vaccines: principles and mechanisms. Science Translational Medicine 10(456): eaat4615.

24. Gootenburg J, Abudayyeh O, Kellner M, Joung J, Collins J, et al. (2018) Multiplexes and portable nucleic acid detection platform with Cas13, Cas12a, and Csm6. Science 360(6387): 439-444.

25. Uppada V, Gokara M, Rasineni G (2018) Diagnosis and therapy with CRISPR advanced CRISPR based tools for point of care diagnostics and early therapies. Gene 656: 22-29.

26. Lu X, Xue H, Ke Z, Chen J, Ji L (2015) CRISPR-Cas9: a new and promising player in gene therapy. Journal of Medical Genetics 52: 289-296.

27. Zhou L, Peng R, Zhang R, Li J (2018) The applications of CRISPR/Cas system in molecular detection. Journal of Cell and Molecular Medicine 22: 5807-5815.

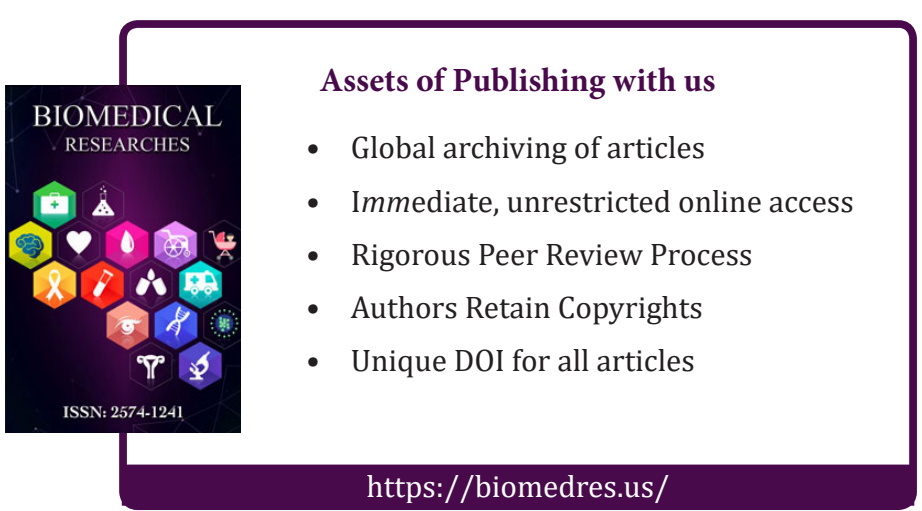

